\title{
CRACKS FORMATION IN NICKEL-BASED SINGLE CRYSTAL ALLOY MANUFACTURED BY SELECTIVE LASER MELTING
}

\author{
${ }^{1}$ Evgenii BORISOV, ${ }^{1}$ Kirill STARIKOV, ${ }^{1}$ Anatoly POPOVICH, ${ }^{1,2}$ Vera POPOVICH \\ ${ }^{1}$ Peter the Great St. Petersburg Polytechnic University, St. Petersburg, Russian Federation, \\ evgenii.borisov@icloud.com \\ ${ }^{2}$ Faculty of Mechanical, Maritime, and Materials Engineering, Delft University of Technology (TU Delft), \\ Mekelweg 2, 2628 CD, Delft, The Netherlands
}

https://doi.org/10.37904/metal.2020.3625

\begin{abstract}
The aim of this study was to determine the influence of selective laser melting (SLM) process parameters on the formation of cracks in nickel-based single crystal alloy. Several sets of laser scanning parameters, with varied laser power, scanning speed and hatch distance, thus resulting in different volumetric and linear energy densities, were investigated. The relationship between energy density, specimen densification, cracks formation and evolution of directional microstructure was shown. It was found that higher linear energy density results in fewer cracking and defects. Cracking in lower energy samples was attributed to the formation of thermal shrinkage pores in the interdendritic spaces and precipitation of $\mathrm{Ta}$, Mo and $\mathrm{Nb}$ carbides along the dendrites boundaries.
\end{abstract}

Keywords: Selective laser melting, Ni single crystal alloys, additive manufacturing, crack formation

\section{INTRODUCTION}

Search for new materials suitable for additive manufacturing (AM) of complex parts is driving the future developments of additive technologies. Recently, there were many scientific papers devoted to the development, characterization and application of new materials in the additive industry [1-6].

Nickel heat-resistant single-crystal silicon-containing alloy was developed in the second half of the last century and is usually used for the production of turbine blades using directional solidification casting method [1]. However, as a result of non-equilibrium solidification conditions, products manufactured by this method are characterized by chemical and structural heterogeneity. The size and shape of the $\gamma^{\prime}$-phase in dendrites and boundary regions differ significantly (in the boundary regions, the $\gamma^{\prime}$-phase particles are much larger than in the axes of dendrites). The use of high-temperature homogenization helps to eliminate heterogeneity and form a more homogeneous microstructure of the alloy. However, it is not possible to eliminate the release of rhenium along the borders of dendrites by heat treatment due to its low diffusive activity. Such rhenium release leads to the formation of topologically densely packed phases.

There exist two negative effects of topologically densely packed phases on the properties of the nickel-based single crystal alloy. Firstly, packed phases are the source of the origin of micro-cracks leading to a decrease in the plasticity of the alloy. Secondly, the absorption of a significant number of $\gamma$-stabilizing elements leads to depletion of the solid solution and reduces the degree of solid-solution hardening.

The advantage of the selective laser melting (SLM) over the directional solidification method is a more homogeneous distribution of alloying elements throughout the entire volume of the product, which will be discussed in this paper. However, the use of SLM requires optimization of process parameters and problems associated to it. One of these problems is in the formation of cracks during the printing process [7-12]. 
Microstructural investigation and cracks analysis in Ni-based specimens manufactured by selective laser melting is described in this paper. The aim of this study is to achieve a deeper understanding of cracking mechanisms in the process of selective laser melting.

\section{EXPERIMENTAL METHODS}

Rectangular specimens were manufactured from nickel alloy powder (the chemical composition is given in Table 1) using Aconity3D MIDI selective laser melting facility (Aconity3D GmbH, Germany). The machine is equipped with laser source with variable focal spot diameter with gas power distribution and a maximum power of $1000 \mathrm{~W}$. Moreover, machine is equipped with a module to enable operation with platform preheating up to $1200^{\circ} \mathrm{C}$. SLM process parameters, resulting in differed values of volume and linear energy density, are shown in Table 2.

Table 1 Chemical composition of Nickel alloy powder used in this study (wt\%)

\begin{tabular}{ccccccccccc}
\hline $\mathrm{Ni}$ & $\mathrm{Cr}$ & $\mathrm{Al}$ & $\mathrm{Mo}$ & $\mathrm{W}$ & $\mathrm{Co}$ & $\mathrm{Re}$ & $\mathrm{Ta}$ & $\mathrm{Nb}$ & $\mathrm{C}$ & $\mathrm{B}$ \\
\hline 62.9 & 4.9 & 5.9 & 1.1 & 8.4 & 9.0 & 1.93 & 4.1 & 1.6 & 0.12 & 0.01 \\
\hline
\end{tabular}

Table 2 Process parameters used in this study

\begin{tabular}{ccccc}
\hline Energy density & Specimen 1 & Specimen 2 & Specimen 3 & Specimen 4 \\
\hline Linear $(\mathrm{J} / \mathrm{mm})$ & 0.24 & 0.45 & 0.6 & 0.8 \\
Volume $\left(\mathrm{J} / \mathrm{mm}^{3}\right)$ & 97 & 97 & 120 & 160 \\
\hline
\end{tabular}

The chemical composition and microstructure of the SLM specimens were analyzed by Carl Zeiss Supra 55VP scanning electron microscope (SEM). The integrity of the sample after SLM was examined on a Leica DMI 5000 light optical microscope.

\section{RESULTS AND DISCUSSION}

Sample's integrity investigated by a Leica DMI 5000 light optical microscope showed that specimens manufactured with higher linear energy density have fewer cracks and fractures. Figure 1 shows panoramic photos of the central part of the specimens along. According to them, specimens 1 and 2 show that the integrity of the material improves with an increase in the value of the linear energy density, despite the same values of the volumetric energy density.
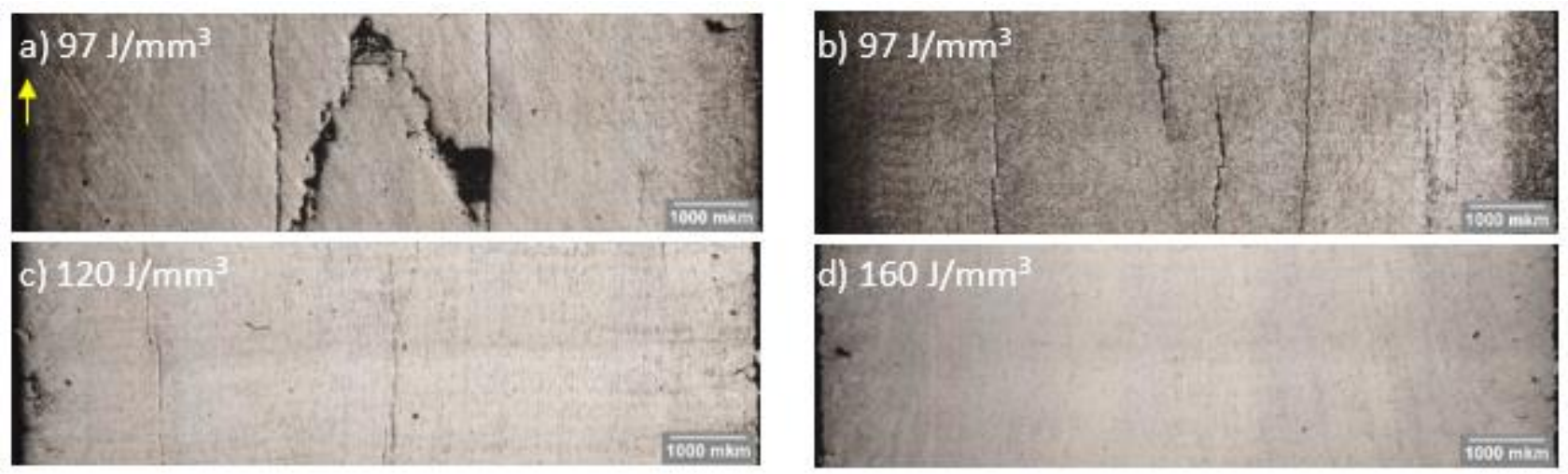

Figure 1 Cracks along the cross sectioned building direction (BD) of the specimen: a) - 1 ; b) - 2; c) - 3; d) - 4 
Table 3 The results of graphical analysis of images obtained with an optical microscope

\begin{tabular}{ccccc}
\hline & Specimen 1 & Specimen 2 & Specimen 3 & Specimen 4 \\
\hline Amount of cracks, \% & $>3.5$ & $>0.8$ & $>0.3$ & $<0.03$ \\
\hline
\end{tabular}

At the same time, photos of specimens 3 and 4 also demonstrate the tendency to reduce the number of defects when using parameters sets that provide higher values of linear energy density. Figure $\mathbf{1} \mathbf{d}$ shows that in sample 4 grown at a linear energy density of 0.8 (Table 2 ). There are practically no large volume failures.

It was found that the obtained specimens have a heterophase structure consisting of columnar $\gamma$-phase cells stretched along the building direction (marked by arrow in Figure 1 ) and highly dispersed $\boldsymbol{\gamma}$-phase based on $\mathrm{Ni}_{3} \mathrm{Al}$ intermetallic along the dendritic boundaries. Moreover, the segregation of alloying element and formation of carbides is observed in the interdendritic zones (Figures $\mathbf{2}$ and $\mathbf{3}$ ). Carbides are important for ensuring the strength properties of heat-resistant nickel-based alloys at temperatures above the solubility limit of the intermetallic $\gamma^{\prime}$-phase. The strengthening effect of carbides is to prevent recrystallization along the borders of dendrites and increase the temperature performance. EDX analysis (Figure 3 ) showed that these precipitations are $\mathrm{Ta}, \mathrm{Mo}$ and $\mathrm{Nb}$ carbides. Figure 3 also shows that the production of parts by selective laser melting contributes to the uniform distribution of rhenium throughout the sample without any additional heat treatment. This observation may indicate the advantage of using the selective laser melting method to produce heat-resistant nickel-based alloys over the traditional directional solidification method.
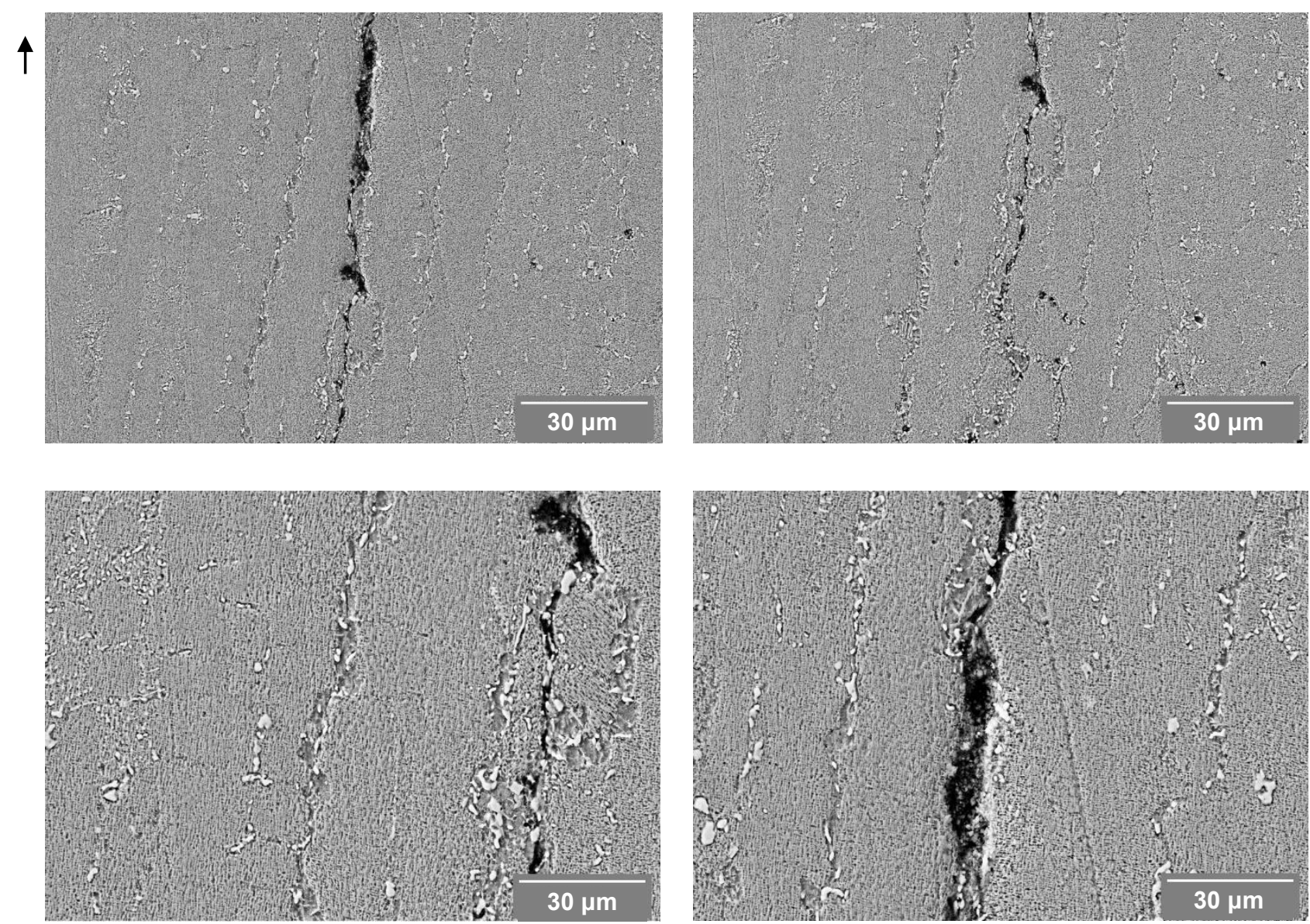

Figure 2 Scanning electron microscopy images showing cracking within dendritic microstructure 
Scanning electron microscopy images showed that cracks are formed exclusively along the boundaries of dendritic grains in places of large accumulation of carbide precipitations, despite the significant role of carbides in the formation of heat-resistant properties of nickel-based alloys.

The crack development mechanism is in the formation of shrinkage micropores in the interdendritic spaces of single crystal alloy and at the boundaries of the $\gamma^{\prime}$-phase and eutectic, and the strengthening $\gamma^{\prime}$-phase particles, as well as $\mathrm{Ta}$, Mo and $\mathrm{Nb}$ carbides.

At high crystallization rates, inherent to the scanning modes with a lower linear energy density, the melt zones are surrounded on one hand by the boundaries of growing dendrites, and on the other by carbides released in the melt. Then, when such zones solidify, thermal shrinkage occurs, which in turn leads to the appearance of crack nuclei. The cracks are formed after the increase in pores volume followed by pores coalesces under the influence of thermal stresses.

It should however be noted that with higher linear energy density, resulting in decreased melt crystallization rate, less of these areas are formed. The process occurs more evenly throughout the entire volume due to the crystallization of the melt since the melt flowing into the necessary zones compensates for the shrinkage of the material, which contributes to the appearance of fewer shrinkage micropores.

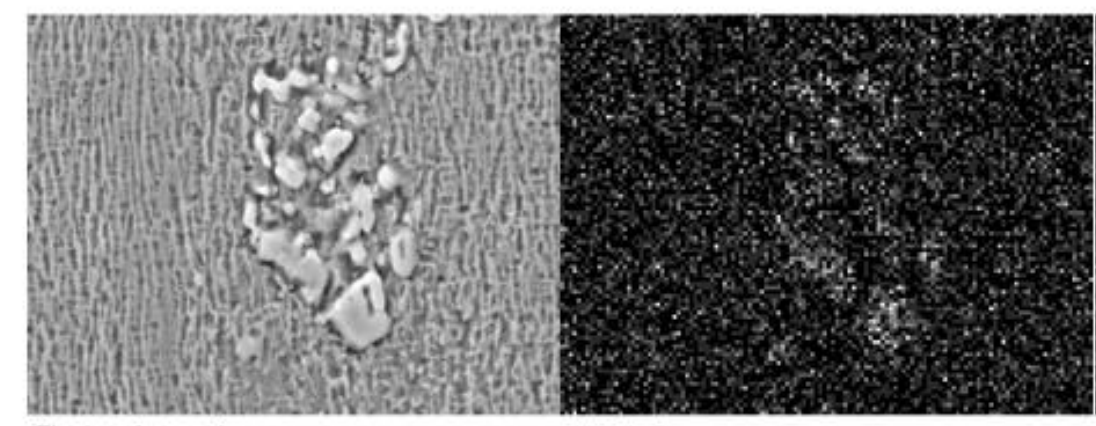

Electron Image 1

CKa1_2

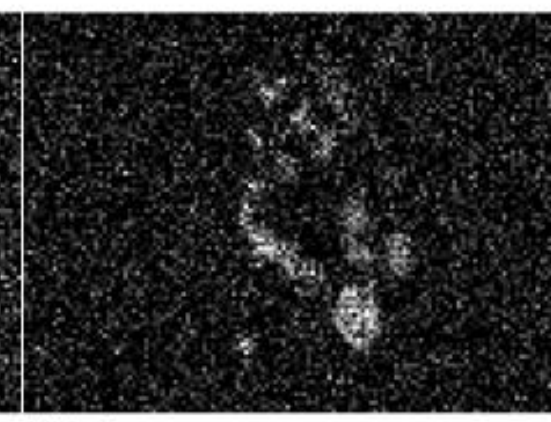

Mo La1

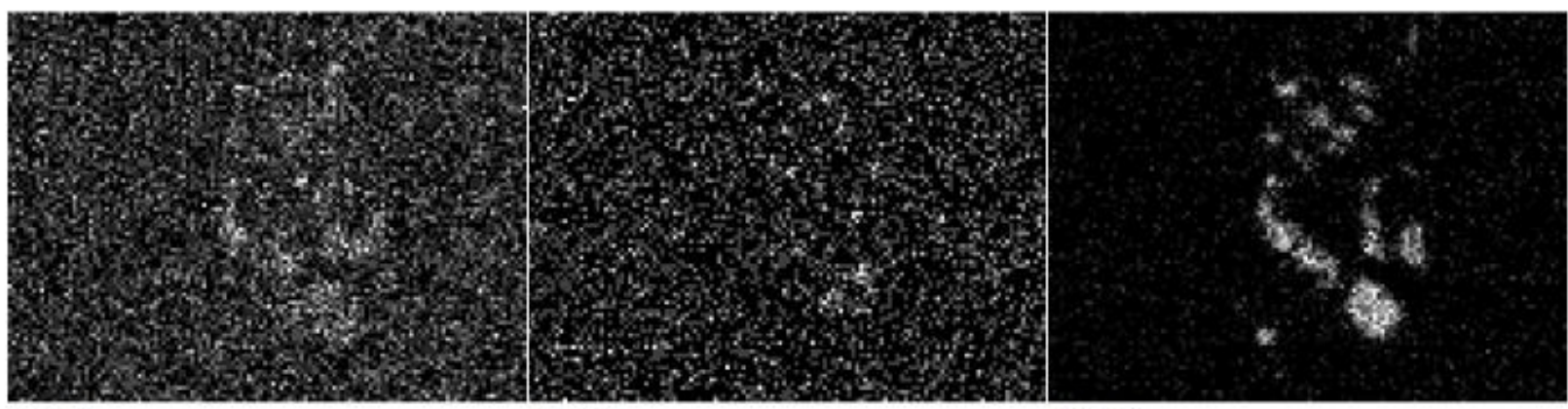

Re La1

Nb La1

Figure 3 Results of EDX analysis of specimens produced by selective laser melting

\section{CONCLUSIONS}

This study shows the influence of various SLM process parameters on the densification and formation of cracks in Ni-based single crystal alloy. It was found that the integrity of the specimens is directly related to the linear energy density. The higher the linear energy density the fewer defects are observed. The mechanism of cracks formation in nickel-based alloys was related to the thermal shrinkage during crystallization of the melt zones surrounded by dendrites on the sides, and by $\mathrm{Ta}, \mathrm{Mo}$ and $\mathrm{Nb}$ carbides. This problem can be solved by reducing the crystallization rate of the entire melt, leading to a more homogeneous solidification, which can be achieved by increasing the linear energy density. Thus the mechanism of crack formation in studied herein nickel-based 
alloy is in the appearance at high crystallization rates of the melt zones inherent in scanning modes with a lower linear energy density, surrounded on the one hand by the boundaries of growing dendrites, on the other hand by carbides released in the melt. Thermal shrinkage occurs when such zones solidify, which in turn leads to the appearance of crack nuclei. However, less of these regions are formed with a decrease in the melt crystallization rate by using scanning modes that provide a higher linear energy density, since the melt crystallization occurs more evenly throughout the volume due to the low speed.

\section{ACKNOWLEDGEMENTS}

This research was supported by Russian Science Foundation grant (project No 19-79-30002).

\section{REFERENCES}

[1] CHAUVET, Edouard et al. Producing Ni-base superalloys single crystal by selective electron beam melting. Scripta Materialia. 2018, vol. 152, pp. 15-19.

[2] POLOZOV, Igor et al. Synthesis of Ti-5Al, Ti-6Al-7Nb, and Ti-22Al-25Nb alloys from elemental powders using powder-bed fusion additive manufacturing. Journal of Alloys and Compounds. 2018, vol. 763, pp. 436-445.

[3] GONCHAROV, I.S. et al. Synthesis of Nb-based powder alloy by mechanical alloying and plasma spheroidization processes for additive manufacturing. Materials Letters. 2019, vol. 245, pp. 188-191.

[4] RAZUMOV, N.G. et al. Morphology of high-strength heat-resistant steel powder for machines for additive production from shavings. Metal Science and Heat Treatment. 2019, vol. 60, no. 1-12, pp. 710-714.

[5] FARBER, E. et al. A review of NiTi shape memory alloy as a smart material produced by additive manufacturing. Materials Today: Proceedings. 2020.

[6] HOSSEINI, E, POPOVICH, V. A. A review of mechanical properties of additively manufactured Inconel 718. Additive Manufacturing. 2019, vol. 30, p. 100877.

[7] XU, Jianjun, et al. The initiation and propagation mechanism of the overlapping zone cracking during laser solid forming of IN-738LC superalloy. Journal of Alloys and Compounds. 2018, vol. 749, pp. 859-870.

[8] KOU, Sindo. A criterion for cracking during solidification. Acta Materialia. 2015, vol. 88, pp. 366-374.

[9] QIU, Chunlei et al. On the solidification behaviour and cracking origin of a nickel-based superalloy during selective laser melting. Materials Characterization. 2019, vol. 148, pp. 330-344.

[10] YANG, Jingjing et al. Cracking behavior and control of Rene 104 superalloy produced by direct laser fabrication. Journal of Materials Processing Technology. 2015, vol. 225, pp. 229-239.

[11] HAN, Quanquan et al. Laser powder bed fusion of Hastelloy X: effects of hot isostatic pressing and the hot cracking mechanism. Materials Science and Engineering A. 2018, vol. 732, pp. 228-239.

[12] LIU, Zhaoyang, HUAN, Qi, LIANG Jiang. Control of crystal orientation and continuous growth through inclination of coaxial nozzle in laser powder deposition of single-crystal superalloy. Journal of Materials Processing Technology. 2016, vol. 230, pp. 177-186. 